\title{
ADAPTACIÓN DE PALLET DE EMBALAJES PARA REUTILIZACIÓN POR EL CLIENTE: UN ENFOQUE EN LA LOGÍSTICA BASADA EN COSTOS COMPARATIVOS
}

José Danilo Haick Tavares (UFPR, Brasil) jdhtavares@gmail.com

Alexandre de Andrade (UFPR, Brasil) alfandrade@yahoo.com.br

Cleibson Aparecido de Almeida (UFPR, Brasil) contato@cleibsonalmeida.blog.br

Resumen: El impacto ambiental, económico y financiero del desarrollo de nuevos productos son indicadores de éxito. Evaluar e indicar estos impactos pueden determinar los límites de recomendación del uso de ese nuevo producto. La reutilización de materiales es una manera de promover la sostenibilidad ambiental y aumentar el ciclo de vida del producto. La paletización, una forma de unitización, proporciona economías de escala en la manipulación de materiales y reduce los costos a lo largo de la cadena de abastecimiento. En este contexto, a partir de un estudio de caso, se comparó el Pallet especial con dimensiones de 1,2 x 1,2 m con el nuevo producto, Pallet, equipado con extensor para mantener las mismas dimensiones. Esta comparación tiene por objeto evaluar el impacto logístico y económico del uso de extensores de Pallets en la unitización de bolsas de papel. Se determinó que la compañía estudio de caso del sector de embalaje en el segmento de bolsas industriales, donde después de la distancia límite de 459 kilómetros extensores de Pallets son económicamente deseables. Por lo tanto, se concluye que el uso de Pallets con extensor después de esa distancia promueve la reducción de costos, así como la sostenibilidad del medio ambiente.

Palabras-Ilave: Paquetes; Paletización; Pallet.

\begin{abstract}
The environmental, economic and financial impacts in the development of new products are performance indicators of success. Evaluate and measure these impacts can determine the boundaries recommendation to use this new product, and identify their potential for success. The reuse of materials is a way to promote environmental sustainability and increase the product life cycle. The Palletizing, a form of unitization, provides economies of scale in material handling costs and decreases costs through the supply chain. In this context, in this case, we compared the special Pallet with dimensions of $1.2 \times 1.2 \mathrm{~m}$ with the new product Pallet with extender to maintain the same dimensions. This comparison aims the logistical and economic impact of the use of extenders in unitization of paper bags Pallets. It was determined that this case study company in packaging sector of the segment of industrial bags, where after the distance limit of 459 kilometers extenders Pallets are economically desirable. Therefore, it is concluded that the use of Pallet extenders after that distance promotes cost reduction and environmental sustainability by reusing materials evaluated among the supply chain.
\end{abstract}

Keywords: Packages; Palletizing; Pallet. 


\section{Introducción}

El manejo de los materiales es considerado como actividad de desperdicio o perdida, según el concepto de lean manufacturing, ya que no agrega valor al producto (MOURA, 1997). Pero, es necesario trasladar los productos, así, el principio más importante de la operación de manejo es buscar reducir la cantidad de operaciones, lo que significa la búsqueda de la mejor manera de reducir el tiempo de manipulación. Este proceso tiene como objetivo mejorar la eficiencia del transporte y disminuir el tiempo de las operaciones de manipulación de los materiales. La unitización debido a paletización en el transporte de cargas hace que sea una ruta crítica para la logística moderna (QINGHUA, 2009).

Así, uno de los objetivos de las organizaciones es reducir los costos a lo largo de la cadena de abastecimiento y la utilización o reutilización de los materiales, es una manera de lograr esta reducción. Por lo tanto se ha tratado de estandarizar las dimensiones del Pallet para facilitar su reutilización a lo largo de la cadena logística. No obstante, la estandarización no es factible para todas las cargas, por lo cual Pallets especiales son desarrollados para resolver casos específicos (BOWERSOX, 2006). Para reutilizar este producto fue desarrollado un Pallet con extensor, el cual provee su reutilización como material intercambiable sin cambiar las dimensiones del caso específico de unitización por la paletización de bolsas industriales de papel.
El objetivo de este artículo es evaluar comparativamente el impacto logístico y económico de la utilización de extensores de Pallets en la unitización de bolsas industriales de papel.

El desarrollo de este trabajo se justifica por los temas ambiental y económico. El primero como una adaptación de los productos para así proporcionar su reutilización, promoviéndose sostenibilidad. El facto de viabilidad económica en la adaptación de Pallet, utilizándose el extensor.

Este trabajo se desarrolló, de acuerdo con Magalhães (2005), “a partir de un análisis documental, que se obtuvo del estudio de caso de una empresa que opera en el sector de embalajes de papel, específicamente bolsas industriales multicapas".

\section{Referencial Teórico}

La gestión de la cadena de abastecimiento introduce la logística para orientación y apoyo de un plan único del flujo de productos (CHRISTOPHER, 2009). En un concepto más simple, las empresas exitosas tienen dos rutas:

- ventaja de costo: asignar un perfil de menor costo, $\mathrm{o}$;

- ventaja de valor: asignar al producto un diferenciador.

Este estudio se ratifica en la base teórica de la industria de embalaje (bolsas industriales de papel) y la unitización por la paletización. 


\subsection{La Industria de Embalajes (Bolsas} de papel)

Las principales motivaciones para el uso de embalajes, según Ballou (2006), son: facilitar el almacenamiento y manipulación para promover una mejor utilización de los equipos de transporte, proteger el producto, promover la venta, cambiar la densidad, facilitar el uso y proporcionar al cliente el valor de reutilización.

Moura (1997), a su vez, define que el propósito del uso de embalaje es proporcionar mejores condiciones para el transporte, almacenamiento, distribución, venta y consumo a un costo total más bajo. Para Bowersox et al. (2010), sus funciones son: protección contra daños, la utilidad con eficiencia y la comunicación.

Para comprender mejor el segmento de embalaje de bolsas, enfoque de este trabajo, es necesario describir el segmento del sector de papel y pulpa celulosa, en el cual se inserta y es configurado por tres sectores: pulpa celulosa, papel y embalaje. Estos en conjunto constituyen la cadena productiva de papel y pulpa celulosa.

La industria de pulpa es compuesta por las empresas productoras de celulosa y la pulpa de alto rendimiento. Esta pulpa puede ser utilizada en la producción de papel. La industria de papel, de acuerdo con Montebello y Bancha (2011), es constituida por las empresas productoras de papeles caracterizados según su propósito: papeles para embalaje, papeles de prensa, papeles de impresión, papeles de escritura, papeles higiénicos, papeles cartón y otros. La industria de embalaje comprende el mercado de bolsas multicapas de papel, segmento de actuación de la empresa estudio de caso.

Las principales funciones de las bolsas de papel son: alojar el producto y protegerlo sin contaminarlo, ser resistente a las condiciones a que sean sometidas, promover la utilización del espacio a lo largo de la cadena de abastecimiento del fabricante al consumidor final (ROBERSON, 1993; JUDICE, 2006).

Los segmentos de negocio son, en orden de relevancia, la construcción civil, alimentos, semillas, farmacéuticos, productos plásticos y productos químicos. La combinación de las propiedades del papel y otros materiales como el polietileno proveen a estos embalajes condiciones diversas a cumplir. El embalaje, que se ilustra en la Fig. 1, permite el relleno y apilado, por medio de equipos manuales o automáticos, proporcionando el manejo más fácil del producto, contemplando la productividad (KLABIN, 2011). 


\section{ReLAInEP}

FIGURA 1 - EJEMPLOS DE BOLSAS DE PAPEL
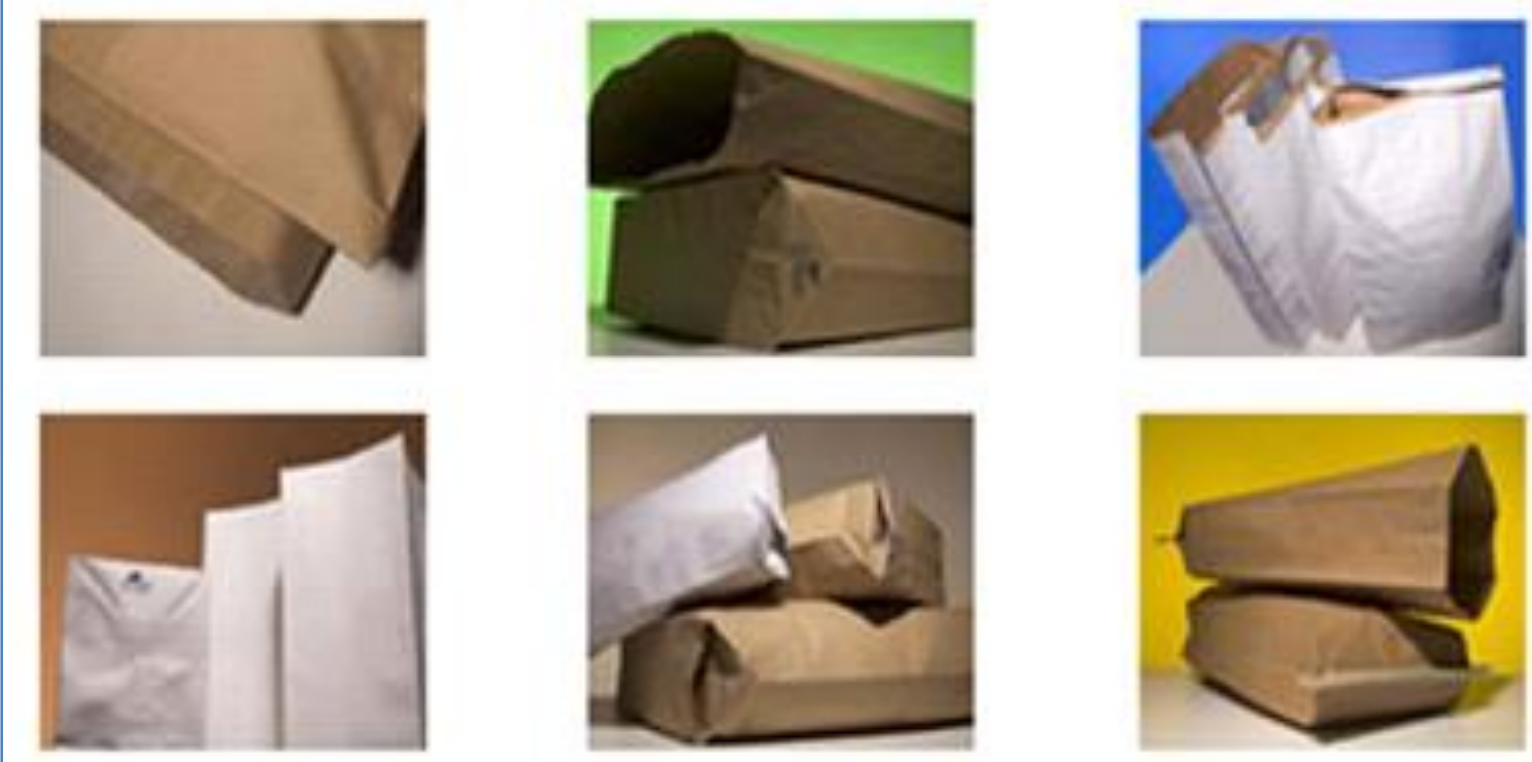

Fuente: Klabin (2011)

\subsection{Paletización}

La paletización es una forma de unitización de cargas. Consiste en el empaque de varios volúmenes de mercancías para formar unidades mayores y así proporcionar la automatización de las operaciones de transporte (SELEME, 2011).

Según Ballou (2006), la unitización promueve economías de escala en la manipulación de los productos. A su vez, Bowersox et al. (2010), añade que además de la eficiencia de manejo, que la unitización ofrece, también incide en la reducción de daños, disminución de robo, reducción de los requisitos de protección previstos por el embalaje, mayor protección contra los factores ambientales, y la unidad de transporte reutilizables que reduce desechos y el descarte del agente de unitización. Por lo tanto, la paletización como forma de unitización promueve estos beneficios $\mathrm{y}$ ventajas. El Pallet, accesorio esencial de la paletización, consiste en una plataforma horizontal de dimensiones específicas (CPP, 1999), donde se deposita la carga apilada, y el cual está diseñado para ser trasladado mecánicamente por grúas, carretillas elevadoras o vehículos de tenedor (MOURA, 1997).

Según Moura (2000), las características y tipos de Pallets de acuerdo con su uso son:

- cautivo: cuyo ciclo de uso se encuentra dentro de los límites de la empresa;

- desechable: será descartado después de un ciclo de uso;

- reutilizable: se utiliza en varios ciclos;

- reversible: sus plataformas superior e inferior son capaces de soportar ciertas cargas; 
- "lo tomas o lo dejas": contesta dos opciones de manipulación, cargando con la carga y cargando la carga sin el Pallet.

Implementar un sistema de transporte por Pallets es establecer un sistema integrado para los usuarios de esta plataforma (QUINGYI, 2003). En este sistema, que se ilustra en la Fig. 2, todos los Pallets tienen especificaciones y estandarizaciones únicas, que ofrecen la posibilidad de intercambiar cuando sea apropiado (QINGHUA, 2009).

La estandarización de los Pallets en el estándar PBR de 1,0 x 1,2 (m) hace que sea económicamente viable mantener el inventario, estaciones de reparación y venta de ese material en la región estandarizada. Formando un pool o sistema integrado de usuarios de Pallets (COSTA, 2002).

Según ABRAPAL (2011), las principales razones de la estandarización de estas medidas $1,2 \times 1,0(\mathrm{~m})$ son:

- la mayoría de las empresas que desarrollan sus actividades en Brasil y usan Pallets tienen sus depósitos adecuados para las dimensiones 1,2 x $1,0(\mathrm{~m})$;

- la medida de 1,2 x 1,0 (m) es la más utilizada en Brasil, Estados Unidos y Europa;

- la forma rectangular ofrece una mayor flexibilidad para la optimización de los vehículos de transporte y el área de almacenaje;

- ningún país utiliza medidas de $1,1 \mathrm{x}$ 1,1 (m) como el estándar de modulación;

- el estándar internacional para las góndolas de supermercados indican que la profundidad máxima es igual a $1,0(\mathrm{~m})$;

- las dimensiones indicadas y definidas por la Internacional Standards Organization son de 1,2 x 1,0 (m); y

- el Estudio del Consejo Económico y Social de las Naciones Unidas (ONU) establece medidas para el high cubic conteiner, teniendo como base los Pallets de 1,2 x 1,0 (m) y 1,2 x $0,8(\mathrm{~m})$. 
FIGURA 2. EJEMPLOS DE UN SISTEMA INTEGRADO DE USUARIO DE PALLETS

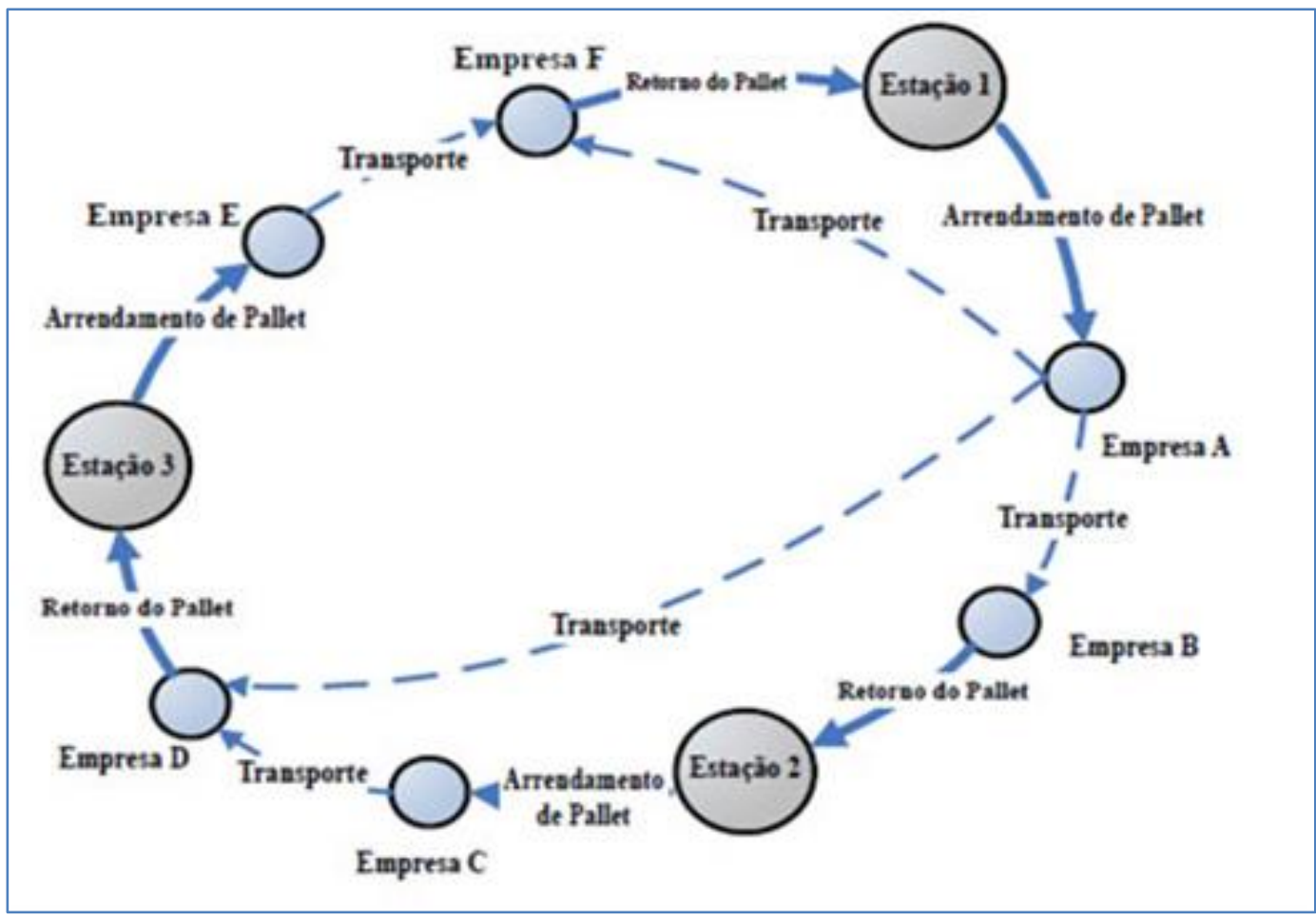

Fuente: Qinghua (2009).

Según Moura (2000), los Pallets de madera pueden estar acompañados de accesorios que permiten el apilamiento de los productos de baja resistencia mecánica y pueden ser desechables, o sea, con bajos costos y menor calidad.

Una carga con unitización, de acuerdo con Ballou (2006), puede aumentar el riesgo de daño si no está correctamente asegurada durante su transporte y manipulación.

Las principales causas de daños, según la empresa objeto de estudio, son cargas ubicadas debajo de Pallets que superan el tamaño del borde y carga de apilamiento inestable. Para Vieira y Roux (2012), las actividades como el transporte de carga de las empresas están obligadas a aceptar la racionalización al máximo de los equipos de almacenamiento y buscar un complemento provocado por la instalación de elementos esenciales para el puerta pallet, pallets desechables o con formato infrecuente. Para evitar estas causas en la paletización de bolsas de papel se utilizan Pallets de anchura y longitud iguales, como puede verse en las Fig. 3 y 4, con dimensiones $1,2 \times 1,2(\mathrm{~m})$ con y sin el extensor. 


\section{ReLAInEP}

FIGURA 3. REPRESENTACIÓN ESQUEMÁTICA DEL PALLET 1,2 X 1,2 M, TIPO TAPA, CON Y SIN EXTENSOR

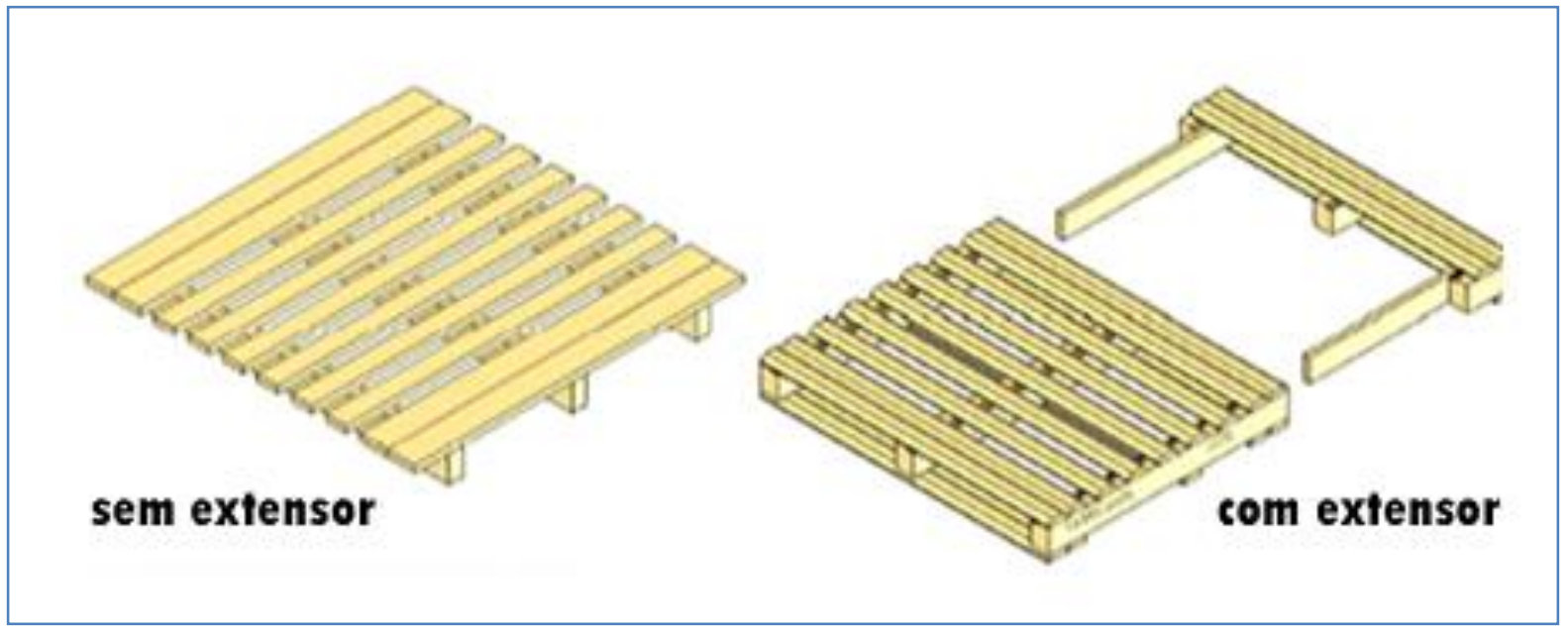

Fuente: Elaborado por los autores (2012)

FIGURA 4. REPRESENTACIÓN ESQUEMÁTICA DEL PALLET 1,2 X 1,2 M, TIPO BASE, CON Y SIN EXTENSOR

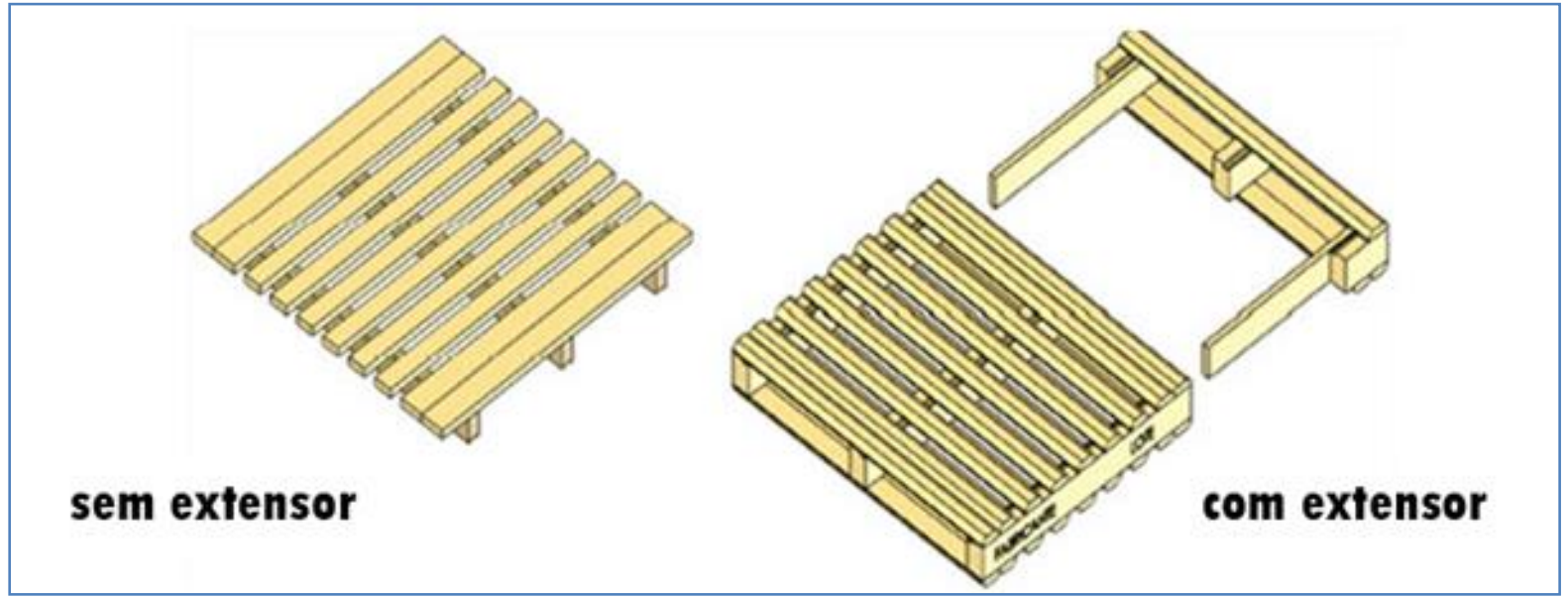

Fuente: Elaborado por los autores (2012)

Los Pallets de las Fig. 3 y 4 están clasificados como especiales, ya que sus dimensiones son diferentes de $1,0 \times 1,2(\mathrm{~m})$, recordando que después de la eliminación del extensor se clasifica como estándar PBR.

\subsection{Trabajos Relacionados}

Puesto que el presente trabajo aborda temas como la modificación, el costo del transporte y la composición de los pallets, esta sección tiene propone presentar algunos trabajos relacionados ya publicados en medios científicos.

En el estudio realizado por Leandro (2006), es posible identificar una diferencia en relación con otros trabajos en la literatura, este nuevo ingrediente fue la preocupación con el medio ambiente, o sea, la sostenibilidad. En este trabajo la autora identifica los factores con el fin de buscar un equilibrio entre dos de los componentes que influencian la sostenibilidad, la cuestión ambiental y la económica. Basado 


\section{ReLAInEP}

en la logística inversa, se realizó el cálculo del coste de los pallets de retorno, los pallets que salen de la empresa A para la empresa B a un destino y, finalmente, regresan a la empresa A. Dado que la empresa B no utiliza los pallets que llegan durante el suministro de sus materias primas, hay una perdida financiera, una vez que los pallets se acumulan en su almacén hasta que se disponen en forma de residuos. Sin embargo, la compañía A ha tenido un costo con la compra de los pallets todas las veces que no vuelvan a la empresa $B$. Con esto, se realizó el cálculo para habilitar el retorno de estos pallets a la empresa de origen de ellos.

Después de los cálculos y la aplicación de los conceptos de la logística inversa para la empresa $A$, ha notado una reducción en el costo con la compra de materias primas para hacer nuevos pallets. La empresa B también he reducido sus costos de almacenamiento $\mathrm{y}$ descomposición de pallets innecesarios para su proceso productivo y todavía colabora con el medio ambiente evitando la eliminación de los materiales existentes en la composición de los pallets. Además, el coste de transporte en el retorno de los pallets ha sido más ventajoso que la fabricación de pallets nuevos.

Por su vez, Hutchins (2011), presentó una patente sobre la invención de un aparato que permite plegar el pallet, como se muestra en la Fig. 5. El autor reporta que esta invención permite almacenar o transportar una cantidad mayor de pallets. El resultado de esto es una economía de espacio dentro de un almacén o un camión, por ejemplo.

FIGURA 5. PALLET FOLDABLE

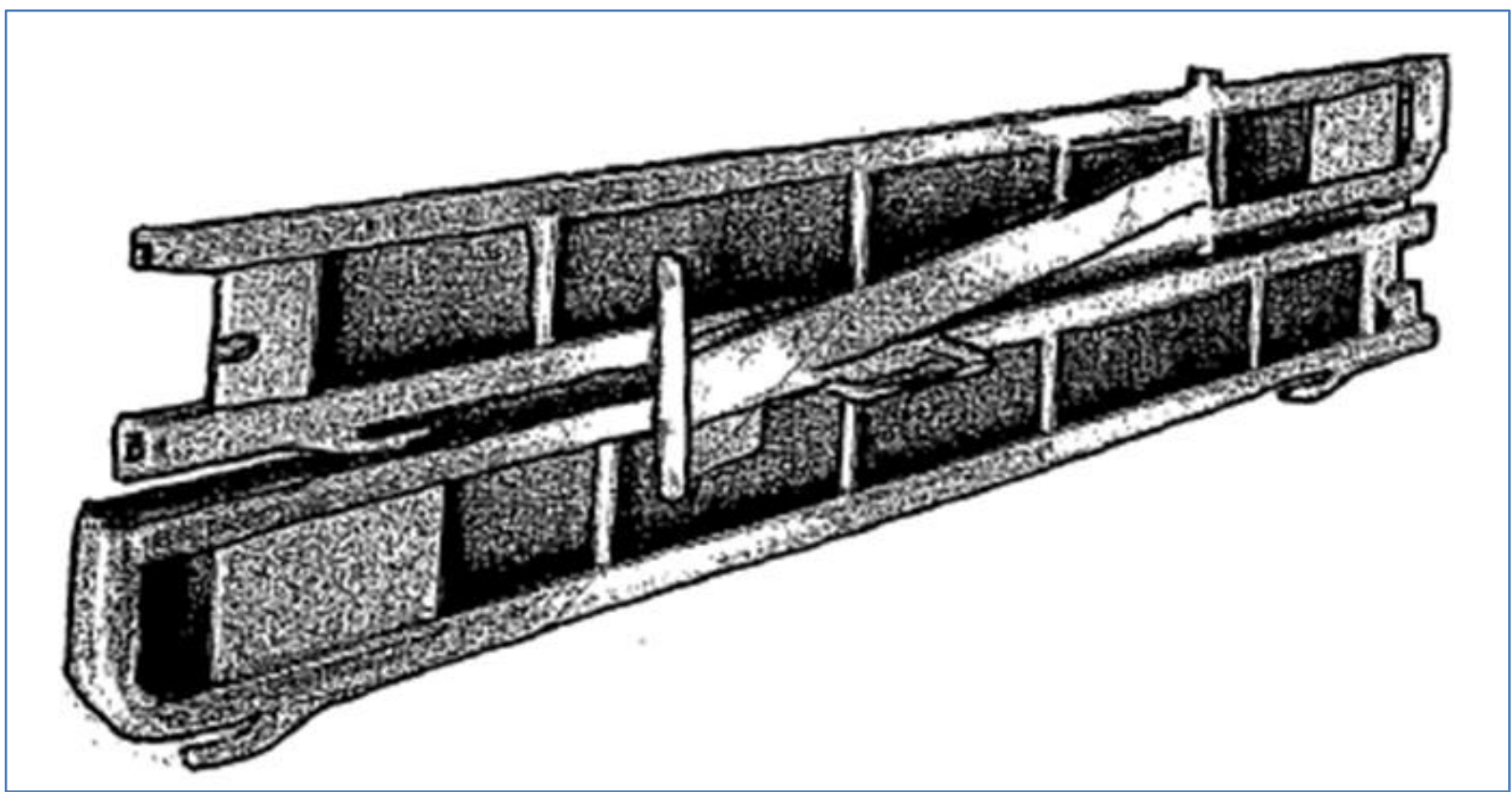

Fuente: Hutchins (2011) 
Técnicamente esta intención considera que un pallet está constituido por una estructura donde las dos bases al lado son fijas y los carriles centrales son móviles. Por lo tanto, desde el momento en que se utiliza el pallet, los carriles centrales son removidos y las bases al lado se unen con la ayuda de un aparato que permite unirse las bases. Se previne que el pallet sea completamente desmontable, asegurándose de que las partes no sean totalmente dispersas después de su uso.

Este modo, el objetivo principal de esta invención es reducir el espacio ocupado por pallets sin carga o vacíos. Uno, Ohuchi y Fujimoto (2012) realizaron una investigación con el objetivo de identificar la mejor composición de los carriles transversales en una plataforma de madera. Para esto, se hicieron cambios en las cantidades de listones de madeira que componen la parte central del pallet. Estas modificaciones fueron hechas considerando 7, 5 y 3 listones transversales, ilustrado en la Fig. 6 (a,b), respectivamente.

Los factores utilizados para evaluar la mejor composición fueron el peso de cada pallet, la cuantidad de materia prima necesaria para la fabricación del pallet, la durabilidad y peso soportado por el pallet.

Después del estudio se comprobó que la mejor configuración del pallet es el modelo con 5 listones de madeira transversales identificado como (b) tipo 7-5 en la Fig. 6. Esta configuración requiere menos materia prima y es más ligero que el modelo con 7 listones de madeira y sostiene el peso equivalente. Además, el modelo con 3 listones de madeira he demostrado ser muy frágil en relación al peso soportado, incluso requiriendo un menor costo para su fabricación.

\section{FIGURA 6. PALLET MODIFICADOS}
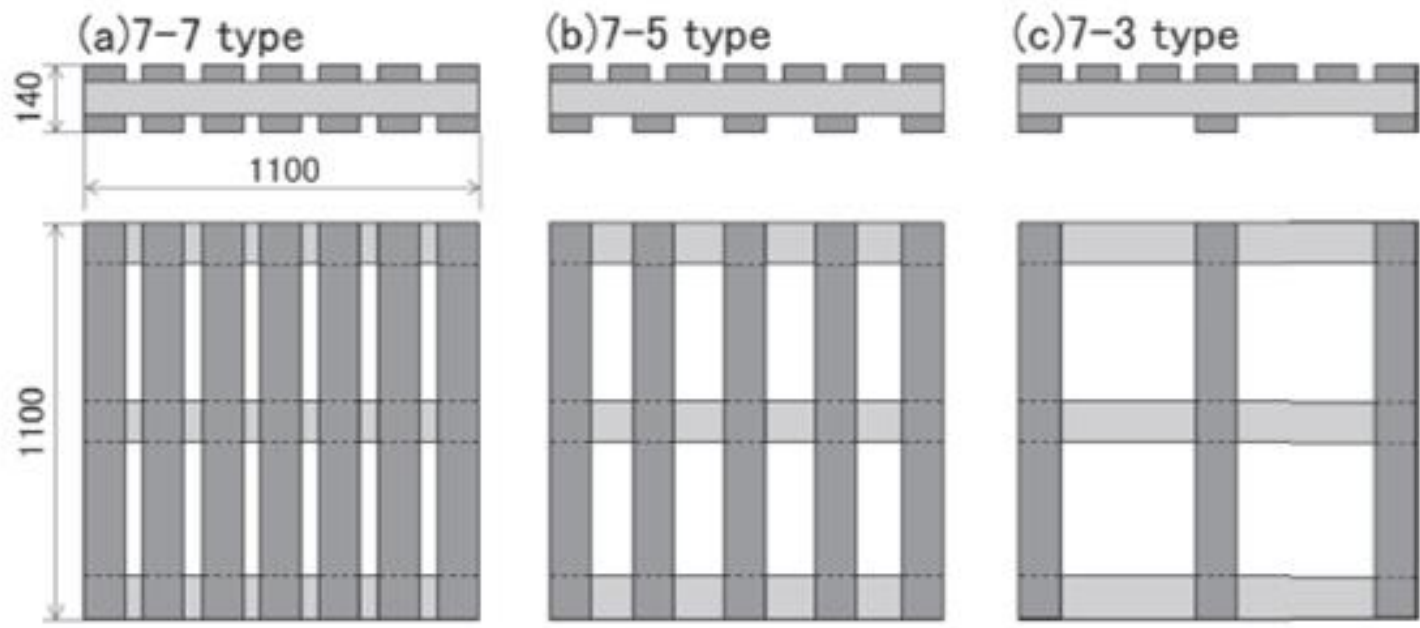

(unit:mm)

Fuente: Uno, Ohuchi y Fujumoto (2012) 


\section{ReLAInEP}

\section{Metodología}

La investigación tiene como contexto el estudio de caso en ambiente organizacional, en la sistematización del análisis de datos, en el diagnóstico, en la observación y la organización de los archivos de la empresa de embalaje.

En primer lugar, se privilegió evaluar la viabilidad económica de un modelo comparativo propuesto en el uso del extensor de Pallet. Mediante la presentación de los resultados empíricos de ensayos anteriores desarrollados por la empresa, se comprobó que, debido a la manera de apilar las bolsas en paquetes debajo del Pallet, es necesario utilizar Pallets con la misma anchura y longitud para atar mejor la carga, proporcionando así la presión entre la tapa y base (Fig. 7), lo que evita el riesgo de daño a la carga durante el transporte y permite apilar y prensar los Pallets cargados. Una particularidad del producto a ser apilado.

El conjunto, que se ilustra en la Fig. 8, de 4005 bolsas paleteadas siempre con la misma carga de servicio por el apilamiento, es usado más frecuentemente por la empresa. En este caso está compuesto de 44 capas, con 6 paquetes por capa y 15 bolsas por paquete. Las capas de la izquierda 1, la principal, y de la derecha la capa 2, la de intermedio. Los colores claros muestran el cuerpo de las bolsas. Los oscuros muestran los extremos donde hay una superposición del cuerpo con el extremo, el color negro identifica las bolsas de la manga. Para completar la cantidad final de bolsas por Pallet son agregados 3 paquetes como capa final.

FIGURA 7. FOTO DEL PALLET BASE/APILADO EN EL CAMIÓN

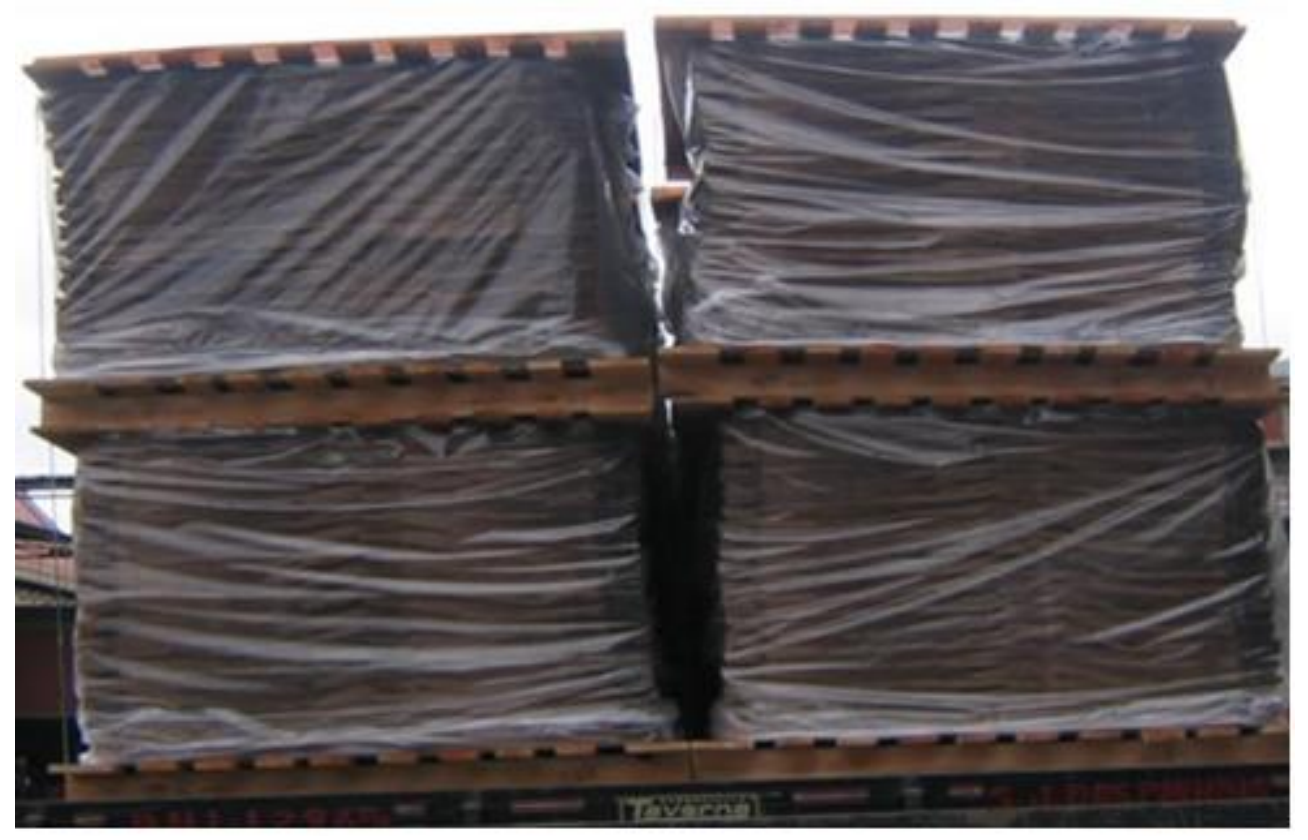

Fuente: Elaborado por los autores (2012) 


\section{ReLAInEP}

FIGURA 8. REPRESENTACIÓN ESQUEMÁTICA DEL APILAMIENTO DE USO EN LA PALETIZACIÓN DE LAS BOLSAS

\begin{tabular}{|c|c|c|}
\hline & \multicolumn{2}{|c|}{ Leyenda } \\
\hline & Cuerpo de la bolsa & $\begin{array}{c}\text { Extremidad de la } \\
\text { primera bolsa } \\
\text { apilada }\end{array}$ \\
\hline & $\begin{array}{c}\text { Extremidad } \\
\text { de la bolsa }\end{array}$ & $\begin{array}{c}\text { Posición de la } \\
\text { válvula } \\
\text { de la bolsa }\end{array}$ \\
\hline $\begin{array}{c}\text { Cuerpo de la } \\
\text { primera } \\
\text { bolsa apilada }\end{array}$ & $\begin{array}{c}\text { Ilustración del } \\
\text { Pallet de madera } \\
\text { Papa } \\
\text { Principal }\end{array}$ & $\begin{array}{c}\text { Capa de } \\
\text { Intermedio }\end{array}$ \\
\hline
\end{tabular}

Fuente: Elaborado por los autores (2012)

\section{Análisis y Discusión de los Resultados}

Identificado el coste de adquisición de Pallets tapa y base con y sin extensor, que se muestra en la Tabla 1. Se observa que el Pallet tapa con extensor tiene un precio de $\$ 14,52$ y el correspondiente de la misma dimensión sin extensor de \$7,13. El Pallet base con extensor tiene un precio de $\$ 14,52$ y el correspondiente de misma dimensión sin extensor de $\$ 7,13$.

Según Reis y Constante (2011) y en común acuerdo con la estrategia de contabilidad gerencial de la empresa en estudio, los costos de transporte se deben sobre todo a la distancia recorrida en el modal y la cantidad del producto enviada. Así se obtiene la ecuación
(1) utilizada para calcular el costo de envío de cada pallet (\$) a su destino basándose en el número de pallets transportados (un), en la distancia recorrida de transporte por carretera (km) y el costo total del transporte de los pallets.

$$
C T K_{u n}=\frac{C T}{Q T * K M}
$$

Así:

CTKun $=$ Costo del transporte de cada Pallet por kilómetro (\$/un*km);

$$
\mathrm{CT}=\text { Costo total del transporte de los }
$$

Pallets (\$);

QT $=$ Cantidad de Pallets transportada (un);

$\mathrm{KM}=$ Distancia en kilómetros recorridos para el transporte $(\mathrm{km})$.

TABLA 1. COSTOS DE ADQUISICIÓN DE LOS PALLETS 1,2 X 1,2 (M)

\begin{tabular}{|l|l|l|}
\hline Dimensión & Tapa (\$) & Base (\$) \\
\hline Pallet 1,2 x 1,2 m con extensor & 14,52 & 14,52 \\
\hline Pallet 1,2 x 1,2 m sin extensor & 7,13 & 7,43 \\
\hline
\end{tabular}

Fuente: Elaborado por los autores (2012) 
A partir de las facturas de los gastos de envío de retorno de los Pallets, fue determinado por la ecuación (1) que el costo del transporte de cada Pallet por kilómetro $(\mathrm{km})$ es de aproximadamente $0,01\left(\$ / \mathrm{un}^{*} \mathrm{~km}\right)$. El costo unitario de transporte para cargar los Pallets se obtiene dividiendo el costo total de envío de $\$ 644,98$ por el número de 77 Pallets $1,2 \times 1,2(\mathrm{~m})$ transportados por $732(\mathrm{~km})$.

Mediante la eliminación del extensor de Pallet sus dimensiones siguen el estándar internacional de 1,0 x 1,2 (m). Por estudios de mercado se determinó que el precio del Pallet estándar fue de aproximadamente $\$ 5,59$ en septiembre de 2011. De acuerdo con la factura de retorno, el costo de la venta del Pallet para el reemplazo es de $\$ 2,79$. En la Tabla 2 se muestran los resultados de los costos de adquisición para el posible regreso después de su uso.

A partir de los datos mostrados en la Tabla 2 se inició una comparación para establecer la diferencia entre el costo neto de los Pallets, con $y$ sin extensor, como se muestra en la Tabla 3 .

\section{TABLA 2. COSTOS DE ADQUISICIÓN DE LOS PALLETS 1,2 X 1,2 (M), RETORNO POTENCIAL DEL CAPITAL POSTERIOR AL USO Y EL COSTO NETO}

\begin{tabular}{|l|l|l|l|}
\hline Dimensión & $\begin{array}{l}\text { Costo de } \\
\text { adquisición }(\$)\end{array}$ & $\begin{array}{l}\text { Retorno } \\
\text { Potencial }(\$)\end{array}$ & $\begin{array}{l}\text { Adquisición - Potencial } \\
\text { Costo neto (\$) }\end{array}$ \\
\hline Pallet $1,2 \times 1,2 \mathrm{~m}$, tapa con extensor & 14,52 & 5,59 & 8,93 \\
\hline Pallet $1,2 \times 1,2 \mathrm{~m}$, base con extensor & 14,52 & 5,59 & 8,93 \\
\hline Pallet $1,2 \times 1,2 \mathrm{~m}$, tapa sin extensor & 7,13 & 2,79 & 4,34 \\
\hline Pallet $1,2 \times 1,2 \mathrm{~m}$, base sin extensor & 7,43 & 2,79 & 4,64 \\
\hline
\end{tabular}

Fuente: Elaborado por los autores (2012)

TABLA 3. DIFERENCIA EN U\$ ENTRE EL COSTO NETO CON Y SIN EXTENSOR

\begin{tabular}{|l|l|l|l|}
\hline & Costo Neto $(\$)$ & $\Delta$ CL $\$$ & \\
\hline Extensor & Con & Sin & Extensor \\
\hline Pallet $1,2 \times 1,2 \mathrm{~m}$ (Tapa) & 8,93 & 4,34 & Pallet $1,2 \times 1,2 \mathrm{~m}$ (Tapa) \\
\hline Pallet $1,2 \times 1,2 \mathrm{~m}$ (Base) & 8,93 & 4,64 & Pallet $1,2 \times 1,2 \mathrm{~m}$ (Base) \\
\hline
\end{tabular}

Fuente: Elaborado por los autores (2012) 


\section{ReLAInEF}

Así se determinó el límite de distancia para que el costo de transporte sea igual a la diferencia de coste entre los Pallets con y sin extensor, de acuerdo con la ecuación (2a).

$$
D L=\frac{\Delta C L}{C T K_{u n}}
$$

Así:

DL $=$ Distancia para equilibrar el costo adicional de fabricación del Pallet con extensor y los costos de transporte $(\mathrm{km})$;

$\Delta \mathrm{CL}=$ Diferencia entre los costos netos que se muestra en la Tabla 3 (\$).

A pesar de la baja frecuencia de reutilización por la empresa, el Pallet sin extensor también es considerado retornable 1 a 2 veces. Es de destacar que la comparación consideró una única reutilización. Substituyendo en la ecuación (2a) el $\Delta \mathrm{CL}$ por el costo neto del Pallet sin extensor tenemos la ecuación (2b), que demuestra económicamente el fundamento de esta reutilización de baja frecuencia.

$$
D L=\frac{C L}{C T K_{u n}}
$$

Así:

DL $=$ Distancia para equilibrar el costo adicional de fabricación del Pallet con extensor y los costos de transporte $(\mathrm{km})$;

$\mathrm{CL}=$ Costo neto del Pallet sin extensor que se muestra en la Tabla 3 (\$).

Conforme la ecuación (2b), se muestra en la Tabla 4, las distancias límites fueron determinadas, lo que demuestra que la no reutilización de Pallets sin extensores es más favorable económicamente para el caso de la unitización de bolsas.

Los datos recogidos muestran que a partir de la distancia de $464(\mathrm{~km})$ no es económicamente inviable volver a utilizar el Pallet tipo base en la unitización de bolsas. El Pallet tapa, a su vez, obtuvo el valor total de $434(\mathrm{~km})$. Recordando que a cada uso, el Pallet sufre algún daño, y por lo tanto reduce su calidad.

TABLA 4. DISTANCIA PARA EQUILIBRAR EL COSTO ADICIONAL DE FABRICACIÓN DEL PALLET SIN EXTENSOR Y EL COSTO DE TRANSPORTE

\begin{tabular}{|l|l|l|l|}
\hline Pallet sin extensor & CL $(\$)$ & $\begin{array}{l}\text { CTKun } \\
(\$ / \mathrm{un} * \mathrm{~km})\end{array}$ & DL $(\mathrm{km})$ \\
\hline Tapa & 4,34 & 0,01 & 434 \\
\hline Base & 4,64 & 0,01 & 464 \\
\hline
\end{tabular}

Fuente: Elaborado por los autores (2012)

TABLA 5. DISTANCIA PARA EQUILIBRAR EL COSTO ADICIONAL DE FABRICACIÓN DEL PALLET CON EXTENSOR Y EL COSTO DE TRANSPORTE

\begin{tabular}{|l|l|l|l|}
\hline Pallet sin extensor & CL $(\$)$ & $\begin{array}{l}\text { CTKun } \\
(\$ / \mathrm{un} * \mathrm{~km})\end{array}$ & DL $(\mathrm{km})$ \\
\hline Tapa & 4,59 & 0,01 & 459 \\
\hline Base & 4,29 & 0,01 & 429 \\
\hline
\end{tabular}

Fuente: Elaborado por los autores (2012) 
Otra advertencia es que para simplificar el estudio se consideró el costo de retiro del extensor igual al de carga y descarga del Pallet sin extensor, esto es lo que se puede desprender de la comparación.

Los resultados de la Tabla 5, obtenidos a partir de la ecuación (2a), demuestran las distancias límites, para lo cual se convierte en, económicamente más favorable, el uso de Pallets con extensores para el caso de unitización de bolsas. Se percibe que después de la distancia de $459(\mathrm{~km})$ es económicamente viable utilizar el extensor en el Pallet tapa en la unitización de bolsas. El Pallet base a su vez obtuvo el valor de 429 (km).

La literatura demuestra, Quinchua (2009), Quingyi (2003), las tendencias en aumentar el número de reutilizaciones y las regiones de estandarización de las dimensiones de los Pallets, promoviendo el fortalecimiento y la expansión del sistema integrado de utilización de Pallet como material intercambiable. Debido a la particularidad de las cargas, Bowersox (2006), todavía Pallets especiales siguen siendo utilizados en algunas aplicaciones. Por lo tanto, desarrollar nuevos Pallets que proporcionen su reutilización, dentro de los estándares dimensionales establecidos, están en línea con esta tendencia y particularidad.

\section{Consideraciones Finales}

La logística está cada vez más consolidada en todas las actividades corporativas. $\mathrm{La}$ competencia entre las cadenas de abastecimiento es real y se puede conseguir el éxito por la correcta administración del manejo de los flujos de materiales e información, o sea, el flujo a lo largo de la cadena logística. Como en la cadena de abastecimiento se reúnen la planificación y la gestión de todas las actividades incluidas en el suministro y la adquisición de mercancías, es importante asegurar la coordinación y la colaboración de todos los involucrados en la cadena de abastecimiento, o sea, proveedores, intermediarios, prestadores de servicios y al mismo tiempo los clientes.

Se requiere el uso de un sistema de unitización de carga capaz de aumentar la productividad de las operaciones, integrando los diversos modos de transporte. Para que podamos tener los productos fluyendo por toda la cadena de abastecimiento con la reducción de coste, sin daño y con excelente nivel de servicio al cliente. Por lo tanto, el Pallet es una manera de unitización que reúne todos los requisitos buscados.

Entre las muchas razones para que ocurran pérdidas financieras con las operaciones logísticas de las empresas, se destaca la falta de control de entrada y salida de Pallets PBR en los centros de distribución y almacenes. Así, se evaluó comparativamente el impacto logístico y económico del uso de extensores de Pallets en la unitización de bolsas. En este sentido, la investigación mostró que en la empresa del estudio, que los extensores son económicamente deseables, o sea, viables para Pallets tipo tapa, ya que reducen los costos a 
lo largo de la cadena de abastecimiento, desde la distancia límite de $459(\mathrm{~km})$, a diferencia del tipo base que representa sólo 93\% del costo neto presentado por lo tapa.

Para ser capaz de demostrar las tendencias de la estandarización y la particularización de los Pallets en la literatura, se recomienda llevar a cabo nuevos trabajos y estudios que analice la reutilización de estos productos y muestren el límite de la viabilidad económica en su aplicación.

\section{Referencias}

$\begin{array}{lrr}\text { ASSOCIAÇÃO } & \text { BRASILEIRA } & \text { DE } \\ \text { FABRICANTES DE PALETES } & \text { PBR - } \\ \text { ABRAPAL. } & \text { Disponível } & \text { em: } \\ \text { <http://www.abrapal.org/index.html>. Acesso } \\ \text { em: 01/10/2011. }\end{array}$

BALLOU, R. H. Gerenciamento da Cadeia de Suprimentos / Logística Empresarial. 5. ed. Porto Alegre: Bookman, 2006.

BOWESOX, D. J. Gestão logística de cadeias de suprimentos. Tradução Camila Teixeira Nakagawa e Gabriela Teixeira Nakagawa. Porto Alegre: Bookman, 2006. 529 p.

BOWESOX, D. J. ; CLOSS, D. J. Logística Empresarial: o processo de integração da cadeia de suprimentos. 9. ed. São Paulo: Atlas, 2010.

COMITE PERMANENTE DE PALETIZAÇÃO - CPP. Especificação para Palete Padrão de Distribuição Nacional PBR. [S.I.] 1999.

COSTA, F. J. C. L. Introdução à administração de materiais em sistemas informatizados. São Paulo: Editora, 2002. p. 80-82.

HUTCHINS, P. Roll pallet apparatus. 2472975, 31 ago. 2011.

JUDICE, J. C. G. Método sistemático para o desenvolvimento da embalagem saco de papel multifoliados para produto em pó ou granulado. Escola de Engenharia de Mauá: São Caetano do Sul, 2006.

KLABIN. Produtora, exportadora e recicladora de papéis do Brasil. Disponível em: <http://www.klabin.com.br/>. Acesso em: 30/05/2011.

LEANDRO, F. Logística Reversa: Percentual de Ocupação dos Paletes na Carga e os Custos de Retorno. Maringá Management: Revista de Ciências Empresariais, Maringá, v. 3, n. 1, p. 17-25, jan/jun 2006.

MAGALHÃES, G. Introdução à metodologia científica: caminhos da ciência e da tecnologia. Editora: Ática, 2005.

MOURA, R. A. Equipamento de movimentação e armazenagem. 5. ed. São Paulo: IMAM, 2000.

MOURA, R. A.; BANZATO, J. M. Embalagem, Unitização e Conteinerização. São Paulo: IMAM, 1997.

MONTEBELLO, A. E. S; BANCHA C. J. C. $\mathrm{O}$ setor de celulose e papel na economia brasileira. O Papel, São Paulo, n. 4, p. 47-50, abr. 2011.

QINGHUA, Z. Pallet Rental Information System Based on RFID. [S.I.]: Logistics and Material Handling, 2009.

QUINGYI, W. Study on Establishing the Pallet Pool System in China. [S.I.]: Logistics and Material Handling, 2003.

REIS, M. A. S.; CONSTANTE, J. M. Metodologia para o Cálculo dos Custos Logísticos Associados ao Fluxo de Mercadorias. São Paulo: FGV, 2011.

ROBERTSON, G.L. Food packaging: Principles and practice. New York: Marcel Dekker, Inc, 1993. p. 676.

SELEME, R. Disciplina de Análises e Operações Logísticas - PPGEP/UFPR. [S.I.]: 2011.

UNO, A.; OHUCHI, T.; FUJIMOTO, N. Studies on Manufacturing of Wooden Flat Pallet by Staggered Connection Using Sugi 


\section{ReLAInEP}

Compressed-dowel and its Process. J. Fac. Agr., Kyushu Univ., Fukuoka, v. 57, n. 1, p. 175-179, 2012.
VIEIRA, Darli R.; ROUX, Miche. Auditoria logística: uma abordagem para operações de centros de distribuição. Rio de Janeiro: Elsevier, 2012. 